\title{
Serotonergic innervation of the rat testis
}

\author{
M. B. Campos, M. L. Vitale*, R. S. Calandra and S. R. Chiocchio* \\ Laboratorio de esteroides, Instituto de Biologia y Medicina Experimental, Obligado 2490, 1428 \\ Buenos Aires, Argentina; and ${ }^{*}$ Instituto de Neurobiologia, Serrano 665, 1414-Buenos Aires, \\ Argentina
}

\begin{abstract}
Summary. The presence of 5-hydroxytryptamine (5-HT) was determined by h.p.l.c. in perchloric extracts of each isolated compartment of the adult rat testis. The testicular capsule, interstitial cells and interstitial fluid contained 5-HT, but 5-HT was not detected in the tubular compartment.

In a group of adult rats, one testis was unilaterally denervated, and the contralateral testis used as control. The superior spermatic nerve, arising from the renal plexus, was excised and 1 week after surgery 5-HT content was measured in the capsule and interstitial fluid of both testes. Denervation caused a significant fall (34\%) in 5-HT content. These results indicate that at least part of the testicular 5-HT derives from a serotonergic innervation of the gonad.
\end{abstract}

Keywords: testis; rat; serotonin; innervation; interstitial fluid; denervation

\section{Introduction}

It has been known since the early reports by Kormano \& Penttilä (1968) that rat testicular tissue contains 5-hydroxytryptamine (5-HT). High concentrations of 5-HT as well as catecholamines and histamine were found in the testes of newborn rats but values were lower in older rats (Zieher et al., 1971).

The administration of high doses of 5-HT causes testicular atrophy, presumably as a result of a severe ischaemia (Boccabella et al., 1962). Effects on endocrine function were observed with implants of smaller amounts of the indolamine (Kinson \& Liu, 1973). On the other hand, direct effects on steroidogenesis have also been reported (Ellis, 1972; Campos et al., 1988) as well as the existence of specific $\left[{ }^{3} \mathrm{H}\right] 5$-HT binding activity in testicular membranes (Campos et al., 1988).

However, the origin of testicular 5-HT and its distribution within the testis remains unexplored. Gonadal 5-HT may originate from serotonergic nerve fibres, uptake from blood, mast cells or intratesticular synthesis.

Histochemical studies (Bell \& McLean, 1973) revealed the presence of fluorescent nerve fibres in the tunica albuginea and tunica vasculosa. This capsular innervation was shown to be mainly adrenergic, with a minor cholinergic component. Nerves were also observed in the mediastinum testis, accompanying blood vessels; but they were never observed near Leydig cells or penetrating seminiferous tubules (Dayan, 1970). It has been suggested that sympathetic innervation may be involved in the regulation of certain testicular functions (Frankel \& Ryan, 1981; Frankel et al., 1984).

The aim of the present study was to investigate the intratesticular distribution of 5-HT and the possible neural origin of the testicular indolamine.

\section{Materials and Methods}

Animals. Adult Sprague-Dawley rats were maintained on a $12 \mathrm{~h}$ light- $12 \mathrm{~h}$ dark schedule in an air-conditioned room $\left(22-23^{\circ} \mathrm{C}\right)$. Food and water were available ad libitum. On the day of the experiment, rats were anaesthetized with ether 
and perfused through the left cardiac ventricle with saline solution $(0.9 \% \mathrm{NaCl}, \mathrm{pH} 7.4)$ containing $10 \mu \mathrm{M}$-pargyline. This monoamine oxidase inhibitor was added to avoid enzymic degradation of 5-HT during perfusion and sample preparation. Testicular interstitial fluid was collected without previous perfusion.

Testicular denervation. Anatomical study of the testicular innervation (Fig. 1) revealed 3 distinct nerve fibre bundles in the superior spermatic nerve. A section of about $10 \mathrm{~mm}$ long was taken from each of these nerves, homogenized in $0 \cdot 1 \mathrm{~N}$-perchloric acid and centrifuged at $12300 \mathrm{~g}$ for $10 \mathrm{~min}$. Then 5 -HT was assayed as described below.

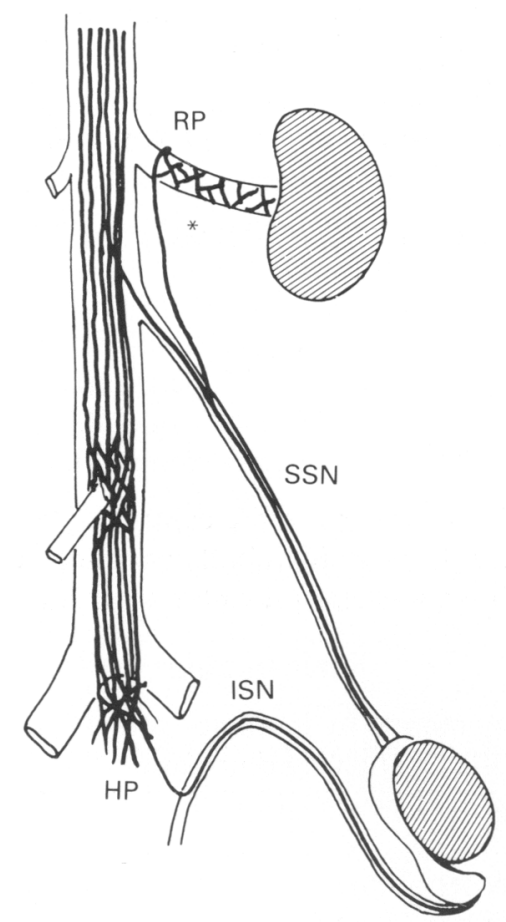

Fig. 1. Schematic diagram of the testicular innervation. SSN: superior spermatic nerves; ISN: inferior spermatic nerves; RP: renal plexus; HP: hypogastric plexus. The asterisk shows the nerve branch which contains 5-HT and which was excised in the denervated group of rats.

To confirm the possibility of a serotonergic innervation of the testis, we measured changes in 5-HT content after the excision of the nerve branch which contained 5-HT (see 'Results'). Testicular denervation was performed unilaterally (left testis) under light ether anaesthesia, and the contralateral gonad served as control. With the aid of a Zeiss dissection microscope the superior spermatic nerve arising from the renal plexus was separated from the spermatic artery and a portion of $5-10 \mathrm{~mm}$ was excised. Undisturbed control animals were used to study normal 5-HT distribution.

Sample preparation. After perfusion, both testes were removed and decapsulated. The testicular capsule was immediately placed on ice, minced and homogenized in $0.1 \mathrm{~N}$-perchloric acid, using glass-glass tissue grinders. Interstitial cells and seminiferous tubules were isolated by mechanical teasing, under a magnifying glass. Interstitial cell viability was greater than $90 \%$ as evaluated by the trypan blue exciusion method. The resulting cell suspension was centrifuged for $10 \mathrm{~min}$ at $800 \mathrm{~g}$, and the pellet was sonicated in $0.1 \mathrm{~N}$-perchloric acid. Isolated tubules were homogenized in the same acid solution using glass-glass tissue grinders. Perchloric acid homogenates of each isolated compartment were centrifuged at $12300 \mathrm{~g}$ for $15 \mathrm{~min}$ and the supernatants were further centrifuged through nitrocellulose microfilters.

Interstitial fluid was collected from individual testes essentially as described by Sharpe \& Cooper (1983). Rats were killed by decapitation and the testes were dissected out. The caudal end of the testicular capsule was carefully incised and the testis placed upright in a polystyrene tube. Fluid was allowed to percolate from the testis into the tube over the next $24 \mathrm{~h}$ at $4^{\circ} \mathrm{C}$. The volume of interstitial fluid was measured and a volume of perchloric acid $0.4 \mathrm{M}$ was added. Contaminating erythrocytes and fluid proteins were eliminated by centrifugation at $12300 \mathrm{~g}$ for $15 \mathrm{~min}$.

Serotonin determination. High performance liquid chromatography was carried out using a LC-4 system (Bioanalytical System Inc., West Lafayette, IN, USA). It was equipped with an Altex pump (110 A model, Altex 
Scientific Inc., Berkeley, CA, USA), a six-port injection valve (Rheodyne, Berkeley, CA, USA) with a $50 \mu \mathrm{l}$ loop, a $10 \mu \mathrm{m} \mathrm{C}_{18}$-uBondapak reverse phase column (Waters, Milford, MA, USA) and an amperometric detector (LC-4, Bioanalytical System Inc.) with a carbon-oil-paste electrode set to a potential of $0.7 \mathrm{~V}$ versus an $\mathrm{Ag} / \mathrm{AgCl}$ reference electrode. The carbon electrode was resurfaced when necessary to ensure high sensitivity. The gain of the detector was set to $10 \mathrm{nA} / \mathrm{V}$.

The mobile phase was prepared with 3-times distilled water (twice distilled with $\mathrm{KMnO}_{4}$ ); it consisted of a $5 \%$ methanol- $0 \cdot 5 \mathrm{M}$-ammonium acetate buffer ( $\mathrm{pH} 5 \cdot 1)$. Before the addition of methanol the buffer was filtered $(0 \cdot 22 \mu \mathrm{m}$ gauze; Milipore, Bedford, MA, USA) and degassed for $2 \mathrm{~h}$ under vacuum. The mobile phase was pumped at a flow rate of $0.9 \mathrm{ml} / \mathrm{min}$ and recycled.

Standard solutions of 5-HT (5-hydroxytryptamine, creatinin sulphate) and 5-HIAA (5-hydroxyindoleacetic acid, dicyclohexylammonium salt) were prepared the day of the experiment, in $0 \cdot 1 \mathrm{~N}$-perchioric acid. Drugs were purchased from Sigma Chemical Co. (St Louis, MO, USA).

Sensitivity of the assay was $100 \mathrm{pg}$; the minimum amount of 5-HT measured in the samples was $300 \mathrm{pg}$.

To determine 5-HT concentrations in plasma, rats were killed by decapitation. Blood was collected and 5-HT assayed as described by Artigas et al. (1985).

Statistics. Statistical analysis was performed by Student's $t$ test.

\section{Results}

\section{5-HT content in testicular compartments}

As shown in Table 1, 5-HT was present in the capsule, interstitial cells and interstitial fluid but not in the tubular compartment. Interstitial fluid contained nanomolar concentrations of the indolamine. The metabolite 5-HIAA was also present in the fluid (data not shown).

Table 1. 5-HT content in rat testicular compartments

\begin{tabular}{lc}
\hline & $5-\mathrm{HT}$ \\
\hline Capsule (ng/mg protein) & $3.4 \pm 0.2$ \\
Interstitial cells (pg/106 cells) & $57.6 \pm 4 \cdot 1$ \\
Interstitial fluid (ng/ml) & $23.3 \pm 0.3$ \\
Seminiferous tubules (ng/mg protein) & $\mathrm{ND}$ \\
Plasma (ng/ml) & $3.9 \pm 2.0$ \\
\hline
\end{tabular}

Values represent mean \pm s.e.m. for 5 individual determinations. $\mathrm{ND}=$ not detectable.

\section{5-HT content in testicular nerves}

5-HT was assayed in a portion of each of the nerve branches of the superior spermatic nerve. These preliminary studies revealed that 5-HT was only present in the nerve bundle arising from the renal plexus (data not shown).

\section{Effect of unilateral denervation on testicular 5-HT content}

5-HT levels in the testicular capsule and interstitial fluid were measured 1 week after unilateral excision of the superior spermatic nerve branch which contained 5-HT. Surgical denervation caused a $34 \%$ decrease in capsular 5-HT content, whereas 5-HT levels in the innervated contralateral capsule remained within control values (Fig. 2a). A similar effect was observed in interstitial fluid 5-HT concentration (Fig. 2b). 


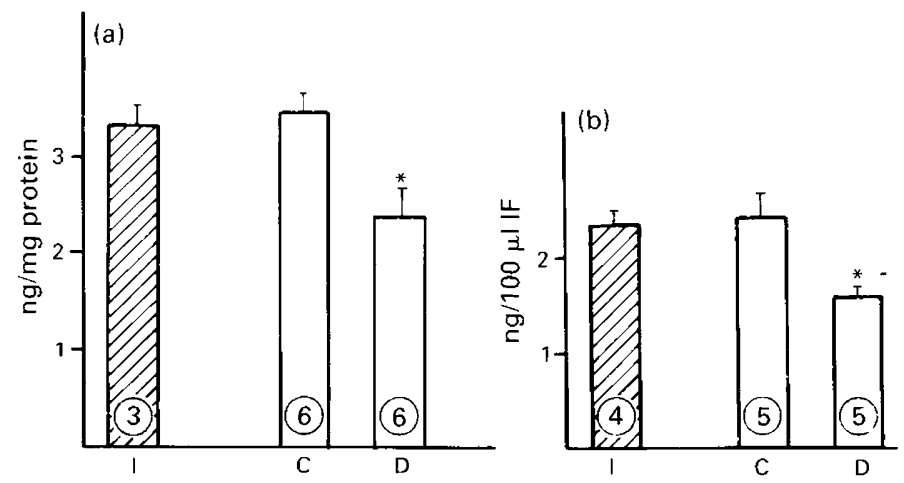

Fig. 2. Effect of unilateral denervation on 5-HT content (a) in the testicular capsule and (b) in the interstitial fluid (IF). I = intact testes of undisturbed rats; $D=$ denervated testes (left); $\mathrm{C}=$ control testes (right) of denervated rats. Values represent mean \pm s.e.m. for the no. of rats indicated. ${ }^{*} P<0.01$ compared with the values for control (C) and intact (I) testes.

\section{Discussion}

The present results show that 5-HT is present in all testicular compartments, except in seminiferous tubules. The high concentration of 5-HT found in the isolated testicular capsule (Table 1) could be related to the presence of fine fluorescent nerve fibres described previously (Bell \& McLean, 1973), since no other fluorescent structures were observed within the capsule. The histochemical technique used (Falck \& Owman, 1965) in these studies identifies all monoamines and therefore serotonergic innervation might also be part of the fluorescent fibres seen in the capsule.

Our results indicate that 5 -HT is present in the interstitial fluid of the adult rat testis. The interstitial fluid receives circulating substances and locally produced factors and provides the medium by which the different compartments communicate (Sharpe, 1984). The 5-HT concentration in interstitial fluid is higher than that in plasma (Table 1). Therefore, capillary filtration does not seem to be the only mechanism accounting for the presence of 5-HT in the interstitial fluid. Alternatively, an appreciable amount could derive from the capsular innervation. The 5-HT concentration in interstitial fluid is in good agreement with the $K^{\mathrm{d}}$ value found for testicular 5-HT binding sites (Campos et al., 1988).

Fluorescent fibres were never seen to end near interstitial cells in the rat. Therefore, the presence of 5-HT in interstitial cell preparations probably reflects the existence of an uptake mechanism which concentrates the indolamine from the surrounding interstitial fluid.

Monoamine oxidase activity has been described in the rat testis, especially located in interstitial cells and walls of seminiferous tubules (Ellis et al., 1972). The same authors suggested that such a distribution provides a protective mechanism in the testis for preventing 5-HT from reaching the germinal epithelium. The presence of 5-HIAA in the interstitial fluid (data not shown) provides further evidence for the existence of testicular monoamine oxidase activity. Moreover, our results demonstrating the absence of 5-HT within the tubular compartment are in good agreement with previous observations.

The specific denervation performed caused a significant decrease in the capsular and interstitial fluid 5-HT content, indicating that serotonergic fibres which arise from the renal plexus and follow the spermatic artery are innervating the testicular capsule. The fact that, after denervation, testicular 5 -HT only fell about $34 \%$ suggests that the excised nerve is not the unique source of testicular 5-HT. Other possible sources could be: (1) different serotonergic fibres which enter the testis through the inferior spermatic nerve (which was not analysed in the present study); (2) the existence of non-serotonergic nerves which take up circulating 5-HT; (3) the presence of mast cells which have 
been described in the tissue surrounding the testicular artery (Sowerbutts et al., 1986) and rarely found in the interstitial space (Pöllänen \& Maddocks, 1988); and (4) the existence of testicular de-novo synthesis of 5-HT, which could be regulated by sympathetic innervation.

Treatment of male rats with human chorionic gonadotrophin induces an increase in testicular interstitial fluid volume (Setchell \& Sharpe, 1981), probably due to an increase in vascular permeability (Sharpe, 1984). Although the cellular mechanism involved in mediating the effect of hCG on the testicular microcirculation is not fully understood, it has been suggested that 5-HT and oestrogens might be involved (Sowerbutts et al., 1986).

The presence of 5-HT binding sites in testicular membranes, and the inhibitory effect of this indolamine on in-vitro testosterone production (Campos et al., 1988), suggest a physiological role of 5-HT in testicular activity.

This work was supported by the Consejo Nacional de Investigaciones Científicas y Técnicas (CONICET), Rep. Argentina.

We thank Mrs Isabel Lopez and Mrs Carmen de Vicenzo for skilful technical assistance.

\section{References}

Artigas, F., Sarrias, M.J., Martinez, E. \& Gelpi, X. (1985) Serotonin in body fluids: characterization of human plasmatic and cerebrospinal fluid pools by means of a new HPLC method. Life Science 37, 441-447.

Bell, C. \& McLean, J.R. (1973) The autonomic innervation of the rat testicular capsule. J. Reprod. Fert. 32, 253-258.

Boccabella, A.V., Salgado, E.D. \& Alger, E.A. (1962) Testicuiar function and histology following serotonin administration. Endocrinology 71, 827-837.

Campos, M.B., Vitale, M.L., Ritta, M.N., Chiocchio, S.R. \& Calandra, R.S. (1988) Involvement of serotonin in testicular androgen production. In Proc. 5th Eur. Workshop on Molecular and Cellular Endocrinology of the Testis, Brighton. p. B16, Cheney \& Sons Ltd, Banbury, Oxon.

Dayan, A.D. (1970) Variation between species in the innervation of intratesticular blood vessels. Experientia 26, 1359-1360.

Ellis, L.C. (1972) Inhibition of testicular androgen synthesis in vitro by melatonin and serotonin. Endocrinology 90, 17-28.

Ellis, L.C., Jaussi, A.W., Baptista, M.W. \& Urry, R.L. (1972) Correlation of age changes in monoamine oxidase activity and androgen synthesis by rat testicular minced and teased preparation in vitro. Endocrinology 90, 1610-1618.

Falck, B. \& Owman, C. (1965) A detailed methodological description of the fluorescence method for the cellular demonstration of biogenic amines. Acta Univ. lund. Sect. II No. 7, 1.

Frankel, A.I. \& Ryan, E.L. (1981) Testicular innervation is necessary for the response of plasma testosterone levels to acute stress. Biol. Reprod. 24, 49l-495.
Frankel, A.I., Mock, E.J. \& Chapman, J.C. (1984) Hypophysectomy and hemivasectomy can inhibit the testicular hemicastration response of the mature rat. Biol. Reprod. 30, 804-808.

Kinson, G.S. \& Liu, C.C. (1973) Testicular response to melatonin and serotonin implanted peripherally in immature rats. Life Science 12, 173-184.

Kormano, M. \& Penttilä, A. (1968) Distribution of endogenous and administered 5-hydroxytryptamine in the rat testis and epididymis. Ann. Med.exp. Biol. Fenn. 46, 468-473.

Pöllänen, P. \& Maddocks, S. (1988) Macrophages, lymphocytes and MHC 11 antigen in the ram and the rat testis. J. Reprod. Fert. 82, 437-445.

Setchell, B.P. \& Sharpe, R.M. (1981) The effect of injected human chorionic gonadotrophin on capillary permeability, extracellular fluid volume and the flow of lymph and blood in the testis of rats. J. Endocr. 91, 245-254.

Sharpe, R.M. (1984) Intratesticular factors controlling testicular function. Biol. Reprod. 30, 29-49.

Sharpe, R.M. \& Cooper, I. (1983) Testicular interstitial fluid as a monitor for changes in the intratesticular environment in the rat. J. Reprod. Fert. 69, 125-135.

Sowerbutts, S.F., Jarvis, L.G. \& Setchell, B.P. (1986) The increase in testicular vascular permeability induced by human chorionic gonadotrophin involves 5 hydroxytryptamine and possibly oestrogens, but not testosterone, prostaglandins, histamine or bradykinin. Aust. J. exp. Med. Sci. 64, 137-147.

Zieher, L.M., Debeljuk, L., Iturriza, F. \& Mancini, R.E. (1971) Biogenic amine concentration in testes of rats at different ages. Endocrinology 83, 351-354.

Received 15 May 1989 